\section{Padrões de aleitamento materno e adequação energética}

\author{
Breastfeeding patterns and energy adequacy
}

\begin{abstract}
To analyze caloric adequacy in infant feeding six months of age or under, the volume of breast milk consumed was estimated through a regression equation as proposed by Drewett 1 . Energy adequacy was estimated according to World Health Organization guidelines for developing countries 2 and the recommended daily allowance of the Food and Nutrition Board 3. A cross-sectional analysis was conducted on data from a cohort of 118 infants in a neighborhood around a health center in Campinas, São Paulo, Brazil, with home interviews by trained students. Data were gathered on social and demographic characteristics, infant feeding patterns, duration of breastfeeding, frequency of feedings, and age when breast milk substitutes were introduced. On average, weaning began early, with a 2.7-month median duration of exclusive breastfeeding. Mean breast milk volume was estimated (from frequency of feeding) as 561.0ml, 558.9ml and $565.5 \mathrm{ml}$ for children in exclusive, predominant, and supplemented breastfeeding, respectively. Mean energy consumption was adequate for all exclusively breastfed children regardless of age and above the recommended level for infants with supplemented breastfeeding and those already weaned.
\end{abstract}

Infant Nutrition; Human Milk; Weaning
Fabíola Figueiredo Nejar 1

Ana Maria Segall-Corrêa 1

Marina Ferreira Rea ${ }^{2}$

Rodrigo Pinheiro de Toledo Vianna 1

Giseli Panigassi 1

\section{Introdução}

Diversos estudos relatam os prejuízos causados às crianças alimentadas precocemente com fórmulas infantis e/ou com leites de outras espécies que não a humana. A substituição do leite materno pode comprometer a saúde da criança, quer nos países desenvolvidos, com a ocorrência de doenças alérgicas e metabólicas, quer nos países em desenvolvimento, com o aumento da morbidade e mortalidade entre os menores de um ano 4,5,6,7.

Os lactentes amamentados exclusivamente com leite materno durante os seis primeiros meses de vida crescem e se desenvolvem adequadamente, além disso, os riscos de infecção do trato gastrintestinal e de alergias ficam diminuídos 8 . Além disso, a interrupção precoce do aleitamento materno exclusivo pode levar à ingestão energética inadequada. Lutter ${ }^{9}$ refere que o consumo energético das crianças em alimentação artificial excede em 15 a 20\% quando comparado ao consumo energético de crianças em aleitamento materno exclusivo. Por outro lado, estudos em populações de países em desenvolvimento mostram que a introdução de papas de frutas para os menores de seis meses que ainda estão sendo amamentados faz reduzir o consumo de leite materno, tendo como conseqüência uma ingestão energética total menor 10,11. A 55a Assembléia Mundial da Organização Mundial da Saúde (OMS) 12 reco- 
menda que o aleitamento materno deve ser mantido de forma exclusiva e em livre demanda até o sexto mês de vida dos lactentes.

As orientações técnicas, que na primeira metade do século passado preconizavam a introdução precoce de alimentos heterólogos, com uso de semi-sólidos e sólidos já a partir de três ou quatro meses, estão hoje superadas. A fisiologia digestiva e o desenvolvimento neuropsicomotor dos lactentes começaram a receber maior atenção, e as práticas alimentares infantis foram progressivamente modificadas 13 .

Conhecimentos mais recentes mostram que a interrupção do aleitamento materno exclusivo em idade inferior a seis meses é inadequada do ponto de vista biológico e psicossocial e representa risco à saúde e bem estar destes lactentes. Segundo Monte \& Sá 14 (p. 2), práticas adequadas de alimentação infantil são aquelas que: "fornecem uma quantidade de alimentos com adequada qualidade para suprir os requerimentos nutricionais; protegem as vias aéreas da criança contra a aspiração de substâncias estranhas e não excedem a capacidade funcional do trato gastrintestinal e rins". Os estágios de maturação do sistema neuromuscular indicam quando e como o alimento pode ser fisicamente manejado pela criança 14,15 .

A adequação energética é relacionada ao consumo alimentar; entretanto, é difícil estimar o consumo energético de bebês amamentados ao peito, já que o volume de leite materno ingerido tem que ser indiretamente mensurado.

Busca-se, no presente estudo, avaliar o padrão de aleitamento materno e adequação energética do consumo alimentar de crianças menores de seis meses, utilizando metodologia validada em estudo populacional 1 .

\section{Metodologia}

Trata-se de um estudo do tipo transversal, realizado por meio de inquérito domiciliar em população residente em bairros da Região Noroeste de Campinas, zona urbana cuja população estudada é de baixa renda familiar, porém com condições satisfatórias de moradia e saneamento. Este trabalho utiliza informações do estudo longitudinal Consumo Alimentar e Saúde 16 que acompanhou por dois anos uma coorte de 454 crianças menores de 12 meses, residentes nesta região, o projeto foi desenvolvido no Departamento de Medicina Preventiva e Social, Faculdade de Ciências Médicas (FCM), Universidade
Estadual de Campinas (UNICAMP), financiado pela Fundação de Amparo à Pesquisa do Estado de São Paulo (no processo - 98/14794-7).

A população geral do bairro estudado era de aproximadamente 20 mil habitantes e 300 nascimentos ao ano. Esperando que $15 \%$ das crianças mantivessem em aleitamento materno exclusivo até o sexto mês e admitindo, além disso, como pior resultado uma prevalência de $5 \%$ de aleitamento materno exclusivo e erro alfa de $5 \%$, calculou-se amostra de 49 crianças. Contudo, prevendo estratificações em três níveis sócio-econômicos, o número de crianças adequado para o estudo seria 147. Este cálculo de amostra para estudo transversal foi feito utilizando o programa Staticalc do Epi Info 6.01. No entanto, tivemos perdas durante o processo de coleta e análise de dados, resultando numa amostra de 118 crianças, sendo este número de crianças suficiente para realização das análises propostas com um poder de teste de $85 \%$.

Sendo assim, selecionaram-se, para este estudo, crianças nascidas entre setembro de 1999 e maio de 2000 que ainda não haviam completado seis meses de vida. Os recém-nascidos foram identificados por intermédio das Declarações de Nascidos Vivos (DNVs) recebidas pelo Centro de Saúde desta área. Cartas foram enviadas previamente com o objetivo de informar o início da coleta de dados. Houve uma recusa de participação. No momento da visita, cada mãe recebeu informações a respeito do trabalho e assinou uma carta de consentimento informado. Esta pesquisa foi aprovada pelo Comitê de Ética em Pesquisa da FCM, UNICAMP.

O questionário da visita domiciliar, semiestruturado, foi preenchido por pessoal treinado durante entrevistas com as mães que duravam em média quarenta minutos. Foram obtidos dados sobre situação sócio-econômica, condições de moradia, característica da amamentação e consumo de alimentos complementares (pelo recordatório alimentar de 24 horas). Outras variáveis coletadas, inclusive medidas antropométricas, não fazem parte da presente análise. Todas as variáveis foram obtidas durante a entrevista. A qualidade da coleta dos dados foi garantida por contínua supervisão das atividades de campo e revisão dos questionários feita pelo coordenador da pesquisa, com cada entrevistador, diariamente, no momento da entrega dos questionários.

Apesar da existência de métodos de estimativas de consumo de leite materno, tais como medidas de peso antes e depois das mamadas 
e uso de solução de deutério para medir ingestão de leite materno 17,18,19, optou-se neste estudo pela utilização da metodologia de Drewett et al. 1, por ter baixo custo, ser de fácil aplicação e não exigir tecnologia. Por este método, estima-se o volume de leite materno consumido pela freqüência ou duração das mamadas, pela quantidade (em quilocalorias) da alimentação complementar e pela idade da criança. Estas são as variáveis incluídas no modelo de regressão linear múltipla proposto pelo autor e utilizado na presente análise.

Modelos de regressão linear múltipla para estimativas de volume de leite materno consumido, com base na duração das mamadas.

- Aleitamento materno exclusivo:

$\mathrm{Y}=591-0.70 \mathrm{X}^{\prime}+0.76 \mathrm{X}^{\prime}$, onde $\mathrm{Y}$ é o preditor do consumo de leite materno, $X^{\prime}$ é a idade em dias e X" é tempo de sucção em minutos.

- Aleitamento materno complementado:

$\mathrm{Y}=755.0-0.48 X^{\prime}-0.59 X^{\prime}$, onde Y é o preditor do consumo de leite materno, $X^{\prime}$ é a idade em dias e X" é o consumo de alimentos complementares em quilocalorias.

Modelos de regressão linear múltipla para estimativas de volume de leite materno consumido, com base no número de mamadas.

$\mathrm{Y}=489-0.63 \mathrm{X}^{\prime}+13.45 \mathrm{X}^{\prime}$, onde $\mathrm{Y}$ é preditor de consumo de leite materno, $X^{\prime}$ é idade em dias e X" é o número de mamadas.

Segundo Drewett et al. 1, o número de mamadas por dia e o consumo de outros alimentos são variáveis que expressam melhor o consumo de leite materno do que apenas a duração das mamadas. O número de mamadas, combinado com a idade do bebê pode oferecer o volume de leite materno consumido.

Para a análise deste estudo utilizaram-se definições dos regimes alimentares diferentes daquelas da OMS 20, exceto para aleitamento materno exclusivo. As categorias alimentares utilizadas neste estudo foram assim definidas:

- Aleitamento materno exclusivo (AMEX): quando o bebê recebia apenas leite materno;

- Aleitamento materno com água e/ou chás (AMAC): quando o bebê recebia leite materno e água e/ou chá;

- Aleitamento materno complementado (AMC): quando o bebê recebia leite materno e qualquer outro alimento além de água e/ou chá; - Desmame: quando o bebê não recebia mais leite materno.

Para adequação energética foi utilizado como referência a Recommended Dietary Allowances (RDA) 3 e as recomendações da OMS 2.

\section{Resultados}

Foram entrevistadas 118 mães com 28,6 anos de idade, em média. A proporção de adolescentes (menor que vinte anos) foi de $11,1 \%$. Cerca de $43 \%$ das mães tinham escolaridade apenas fundamental ou nenhuma, não se observando diferenças significativas com relação à escolaridade dos pais (Tabela 1).

A renda familiar mensal média foi de US\$ 231,76 (DP = US\$ 205,52) sendo a mediana relativamente baixa (US\$152,19). Praticamente $100 \%$ das famílias têm acesso à rede pública de esgoto, água encanada e condições de moradia que, de uma forma geral, podem ser consideradas adequadas.

Das 118 crianças, 97 (82,2\%) ainda mamavam no peito. Estavam em aleitamento materno exclusivo $27,1 \%$, o restante já com alimentação complementar. Vale ressaltar a diminuição do número de crianças em aleitamento materno exclusivo de uma faixa etária para outra. Até 2 meses 36,4\%, entre 2 a 4 meses 35,8\%, caindo esta prática para $11,6 \%$ na faixa etária de 4 a 6 meses (Figura 1). A mediana de aleitamento materno exclusivo encontrada foi de 2,7 meses.

Tabela 1

Distribuição das crianças, segundo características sócio-demográficas. Campinas, São Paulo, Brasil, 1999.

\begin{tabular}{|c|c|c|}
\hline Características & $\mathbf{n}$ & $\%$ \\
\hline \multicolumn{3}{|l|}{ Idade materna (anos)* } \\
\hline 15 a 19 & 13 & 11,1 \\
\hline 20 a 29 & 52 & 44,4 \\
\hline Mais de 30 & 52 & 44,4 \\
\hline \multicolumn{3}{|l|}{ Escolaridade da mãe** } \\
\hline Nenhuma & 2 & 1,7 \\
\hline Fundamental (1a a 8ạ série) & 48 & 41,0 \\
\hline Médio & 46 & 39,3 \\
\hline Superior & 21 & 18,0 \\
\hline \multicolumn{3}{|l|}{ Escolaridade do pai ${ }^{\star \star \star}$} \\
\hline Nenhuma & 2 & 2,0 \\
\hline Fundamental (1a a 8a série) & 38 & 38,0 \\
\hline Médio & 37 & 37,0 \\
\hline Superior & 20 & 20,0 \\
\hline Não sabe & 2 & 2,0 \\
\hline Não respondeu & 1 & 1,0 \\
\hline Total & 118 & 100,0 \\
\hline
\end{tabular}

* 1 dado em branco

** 1 dado não sabe

*** 18 dados não lembra 
O processo de desmame nesta população acontece de maneira esperada, ou seja, a proporção aumenta conforme aumenta a faixa etária, chegando a cerca de $30 \%$ a proporção de crianças com desmame completado entre 4 e 6 meses de idade.

Notou-se, também (Tabela 2), tendência de redução do tempo de mamada à medida que aumentava a idade, além de valores superiores de duração das mamadas entre as meninas. Todavia, não foram verificadas diferenças estatisticamente significativas na freqüência diária de mamadas, entre os gêneros ou nas diferentes faixas etárias.

Valores médios de freqüência e duração das mamadas estão descritos na Tabela 3. Observou-se que a freqüência das mamadas foi reduzida em cerca de um terço quando a criança passava a receber alimentos complementares tais como, outro leite, alimentos semi-sólidos e sucos.

A estimativa de consumo de leite para aleitamento materno exclusivo é maior quando se utiliza para seu cálculo a duração de cada mamada ao invés da freqüência diária das mamadas, sendo a diferença de 106,9ml de leite ou $74,8 \mathrm{kcal}$. O mesmo acontece para cálculo de consumo de leite materno para crianças em aleitamento materno com adição de água e chá e aleitamento materno complementado, isto é, o cálculo a partir do número de mamadas resulta em volume menor de leite ingerido (diferença de $129,29 \mathrm{ml}$ ou $90,50 \mathrm{kcal}$ e $47,55 \mathrm{ml}$ ou $33,29 \mathrm{kcal})$.

Independentemente da opção do método para estimativas, o volume de leite materno é sempre superior quando a criança está em AMEX ou AMAC. Conseqüentemente os valores calóricos obtidos são também superiores, no regime de aleitamento materno exclusivo e com adição de água e chá. Crianças em desmame completo, entretanto, apresentam consumo energético maior. As Figuras 2 e 3 ilustram as tendências até aqui descritas.

O consumo energético das crianças em aleitamento materno exclusivo é inferior ao das demais em outros regimes dietéticos, embora o consumo de leite materno seja superior como descrito anteriormente.

Utilizando a RDA 3 como referência para a análise de adequação, observa-se que os bebês em aleitamento materno exclusivo e aleitamento materno complementado têm déficits de $28,0 \%$ e $11,0 \%$ respectivamente, enquanto que crianças desmamadas apresentam superadequação $(28,4 \%)$.
Figura 1

Distribuição em porcentagem das crianças, segundo alimentação e faixa etária. Campinas, São Paulo, Brasil, 1999.

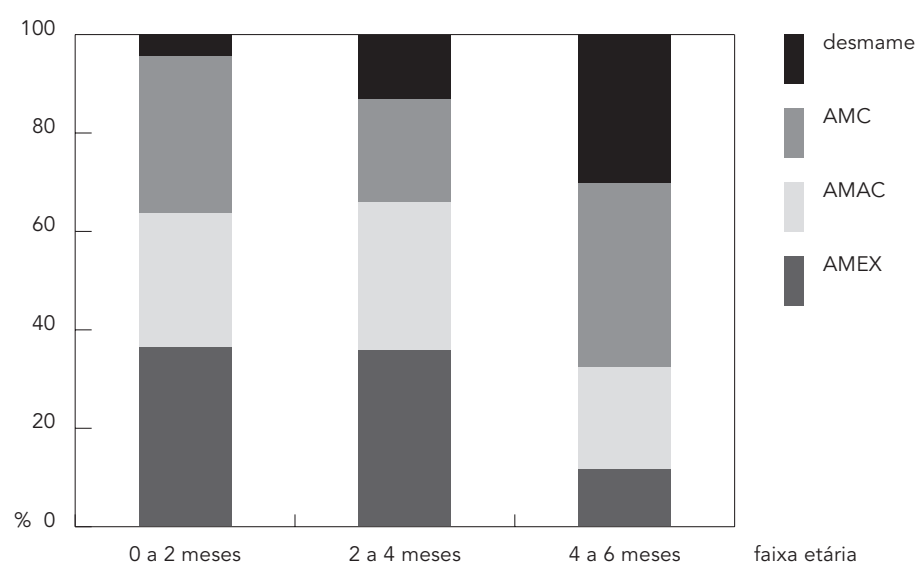

AMEX = aleitamento materno exclusivo; $A M A C=$ aleitamento materno com água e/ou chás; $\mathrm{AMC}=$ aleitamento materno complementado.

\section{Discussão}

Este estudo abordou uma população estável, morando em condições ambientais adequadas, com renda e escolaridade dos pais superiores nas médias do Estado de São Paulo 21. A análise da coorte estudada evidenciou condições nutricionais também satisfatórias com apenas $1,8 \%$ das crianças apresentando adequação de peso para idade inferior a menos 2 escore $\mathrm{z}$ mediana, em relação à da população de referência 22 .

As práticas de amamentação das crianças estudadas não diferem muito, apesar de mostrarem duração pouco superior nas diferentes modalidades de aleitamento materno, daquelas encontradas em outras populações urbanas do Brasil.

A pesquisa sobre a "Prevalência do Aleitamento Materno nas Capitais Brasileiras e Distrito Federal”, realizada em 1999, mostra que a maioria das crianças é amamentada no primeiro mês de vida (freqüência máxima e mínima de $98,3 \%$ e $88,2 \%$, respectivamente), e que a redução desta prática ocorre moderadamente ao longo dos meses, de modo que aos seis meses os valores máximos para a região sudeste são de $84 \%$ 15. Comparada ao estudo clássico da Pesquisa Nacional sobre Demografia e Saúde (PNSN) / Demographic and Health Surveys (DHS), a duração do aleitamento materno exclusivo entre 
Média de freqüência e tempo de mamadas (em 24 horas), segundo faixa etária e gênero. Campinas, São Paulo, Brasil, 1999

\begin{tabular}{|c|c|c|c|c|c|c|c|c|}
\hline \multirow{3}{*}{$\begin{array}{l}\text { Faixa etária } \\
\text { (meses) }\end{array}$} & \multicolumn{4}{|c|}{ Freqüência média de mamadas (DP) } & \multicolumn{4}{|c|}{ Tempo médio da mamada em minutos (DP) } \\
\hline & \multicolumn{2}{|c|}{ Masculino } & \multicolumn{2}{|c|}{ Feminino } & \multicolumn{2}{|c|}{ Masculino } & \multicolumn{2}{|c|}{ Feminino } \\
\hline & $\mathrm{n}$ & & $\mathrm{n}$ & & $\mathrm{n}$ & & $\mathrm{n}$ & \\
\hline De $0 \vdash 2$ & $7 *$ & $8,00(2,83)$ & 13 & $9,92(5,52)$ & $7 *$ & $174,14(93,29)$ & 13 & $217,42(115,18)$ \\
\hline De $2 \vdash 4$ & 21 & $9,52(5,04)$ & 25 & $9,20(3,91)$ & 21 & $200,45(181,94)$ & 25 & $195,12(201,28)$ \\
\hline De $4 \vdash 6$ & 19 & $6,84(2,50)$ & 11 & $8,32(4,24)$ & 19 & $110,26(54,51)$ & 11 & $195,23(131,87)$ \\
\hline
\end{tabular}

* 1 criança sem dado de tempo de mamada

AMEX = aleitamento materno exclusivo; $A M A C=$ aleitamento materno com água e/ou chás; $A M C=$ aleitamento materno complementado.

Tabela 3

Média do número de mamadas e tempo de mamada (em minutos) nas últimas 24 horas, segundo categoria do aleitamento materno. Campinas, São Paulo, Brasil, 1999.

\begin{tabular}{lccc}
\hline $\begin{array}{l}\text { Categoria aleita- } \\
\text { mento materno }\end{array}$ & $\mathbf{n}$ & $\begin{array}{l}\text { Média da freqüência } \\
\text { das mamadas (DP) }\end{array}$ & $\begin{array}{l}\text { Média do tempo de } \\
\text { mamadas (minutos) (DP) }\end{array}$ \\
\hline AMEX & $31^{*}$ & $9,44(4,20)$ & $182,55(150,09)$ \\
AMAC & 31 & $9,77(4,36)$ & $239,37(189,42)$ \\
AMC & 34 & $7,21(3,69)$ & $126,35(85,56)$
\end{tabular}

* 1 criança sem dado de tempo de mamada.

AMEX = aleitamento materno exclusivo; $\mathrm{AMAC}=$ aleitamento materno

com água e/ou chás; $A M C$ = aleitamento materno complementado.

Figura 2

Proporção do consumo energético total ingerido de acordo com categoria alimentar. Campinas, São Paulo, Brasil, 1999.

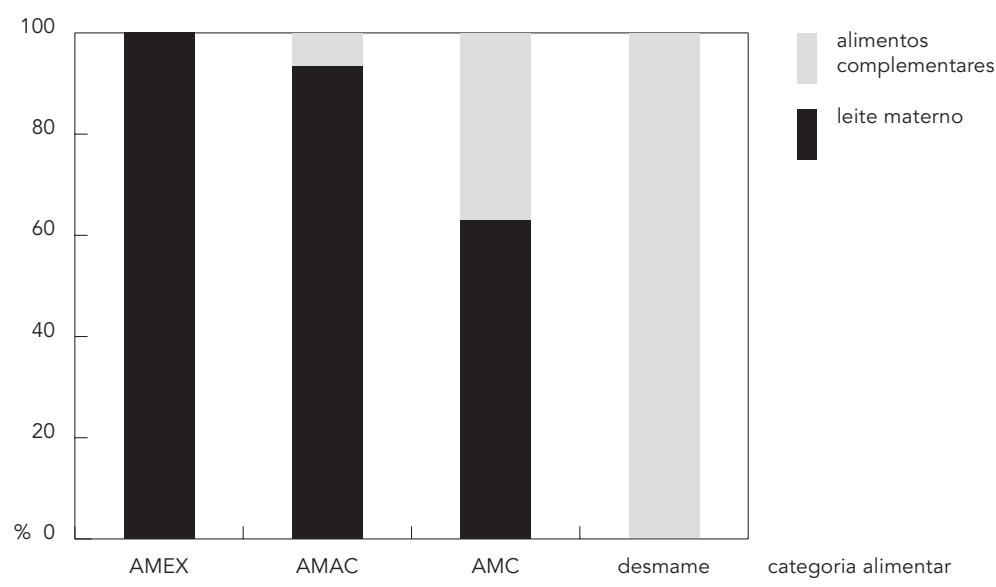

AMEX = aleitamento materno exclusivo; $A M A C=$ aleitamento materno com água e/ou chás; $\mathrm{AMC}=$ aleitamento materno complementado. as crianças aqui estudadas é superior (82 dias) a maior mediana brasileira que é em São Paulo (57 dias) 15.

Com base nos resultados obtidos nesta investigação, pode-se dizer que a freqüência de crianças que iniciam a amamentação é relativamente baixa, a saber, $82 \%$. Essa proporção encontra-se abaixo dos outros estudos, que nesta mesma faixa etária observam percentuais maiores: na PNDS 23 a proporção das mães que iniciam a amamentação foi de $97 \%$ e na PNDS/ DHS 24 de 92\%; porém, estes últimos referemse a informações retrospectivas coletadas entre mães de crianças menores de cinco anos.

Mesmo com estas observações, a prática de aleitamento materno nesta população está longe do preconizado pela OMS, mostrando inadequação importante ao se analisar ao longo das idades.

A proporção de crianças amamentadas diminuiu abruptamente entre as duas faixas etárias ( 0 e 2 meses e 2,1 e 4 meses de idade), caracterizando processo de desmame precoce e rápido. Entre as crianças menores de 2 meses, $36,4 \%$ estavam em AMEX na faixa etária de 2 a 4 meses, houve uma diminuição de $1 \%$, mas entre as idades de 4 e 6 meses a intensidade da queda foi muito maior $(23,8 \%)$, vale dizer, apenas $11,4 \%$ das crianças recebiam exclusivamente leite materno.

Esta queda do 4o mês para o 6o mês pode estar associada à recomendação da OMS que preconizava até recentemente leite materno exclusivo por 4 ou 6 meses de vida do bebê e não até o sexto mês como diz a nova recomendação. O conflito entre uma recomendação e outra tem sido, desde muito tempo fruto de interesses comerciais de produtores de alimentos utilizados por lactentes, que lucram mais quando seus produtos são recomendados a partir do quarto mês. Estes interesses não devem sobrepujar os interesses da saúde pública 25 . 


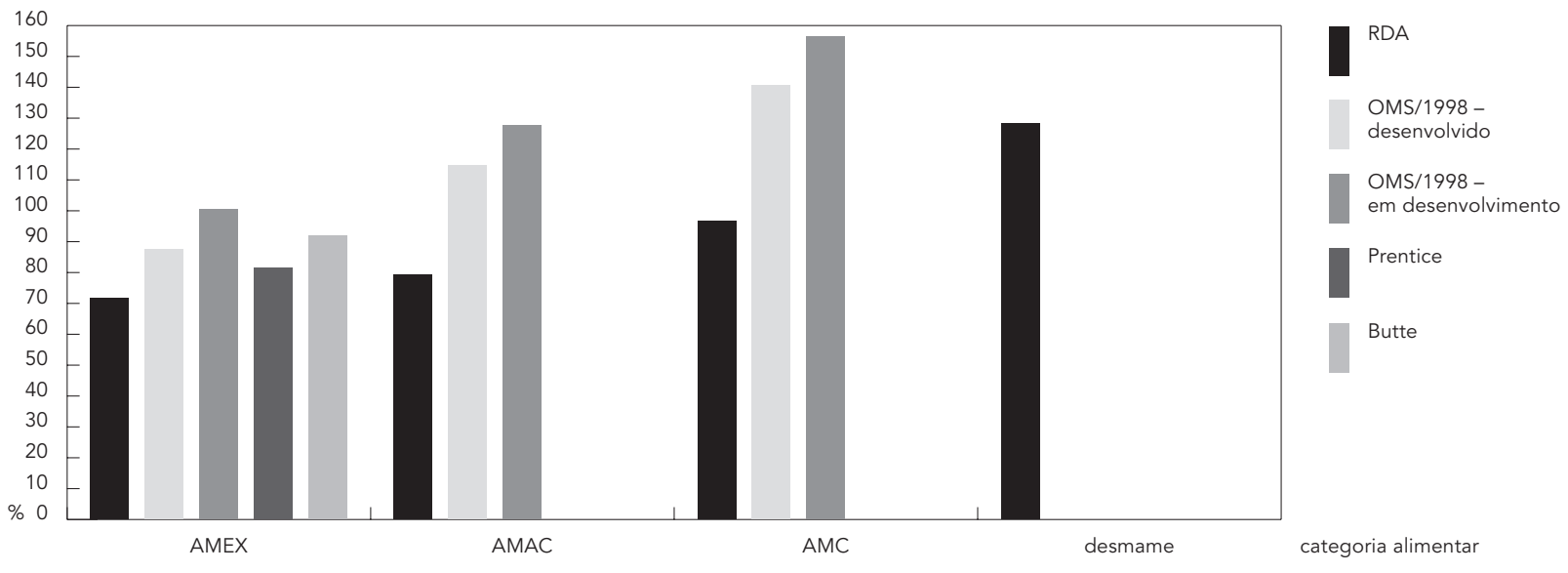

AMEX = aleitamento materno exclusivo; $A M A C$ = aleitamento materno com água e/ou chás $\mathrm{AMC}=$ aleitamento materno complementado.

Observou-se que o consumo energético das crianças em aleitamento materno exclusivo é adequado quando a referência é aquela recomendada pela OMS 2 , para países em desenvolvimento. Nestes países a prevalência de crianças em amamentação exclusiva é ainda superior à dos países industrializados.

São as diferenças observadas entre países desenvolvidos e em desenvolvimento quanto à prática de amamentação que tornam as recomendações da RDA 3, baseadas em crianças com baixa taxa de amamentação, inadequadas para comparação com este estudo, salvo quando se analisam crianças em desmame completo.

Verificaram-se tendências de freqüência e duração das mamadas maiores entre crianças mais jovens e do sexo feminino. Duração e freqüência das mamadas foram maiores em situação de aleitamento materno exclusivo e aleitamento materno com água e/ou chá, quando comparados com o aleitamento materno complementado.

A freqüência observada das mamadas foi semelhante à encontrada por outros autores. Carvalho et al. 26 e Dewey et al. 27 relatam freqüência de dez mamadas em 24 horas, no entanto, Imong et al. 18 encontraram média superior a 15,2 vezes. Com relação ao tempo de mamada, todos os estudos citados acima tiveram média inferior, sendo, 162 26, 14527 e 15118 minutos. As tendências de freqüência e duração das mamadas maiores entre crianças mais jovens e do sexo feminino também são relatadas por Carvalho et al. 26 e Dewey et al. 27.
A média de energia consumida pelos bebês em AMEX foi de 467,55kcal/dia; crianças em AMAC e AMC tiveram consumo energético de $481,75 \mathrm{kcal}$ e $395,89 \mathrm{kcal}$, respectivamente. O consumo de energia dos bebês em AMEX é inferior ao dos bebês em AMAC porque a média de idade dos bebês em AMEX é menor (87 dias contra 98 dias de idade dos bebês em AMAC), e a média do tempo de mamada também é inferior par bebês em AMEX.

Os valores de consumo energético são inferiores ao recomendado pela RDA 3 . Entretanto, sabe-se que a RDA tem valores superestimados, muito provavelmente porque são baseados em população infantil da década de 80 , com baixa prevalência de amamentação e alto consumo de fórmulas infantis hipercalóricas $2,13,28$. O consumo energético no grupo estudado apresenta-se adequado, quando a referência usada é a da OMS para países em desenvolvimento ${ }^{2}$.

Porém, o padrão indicado de adequação energética da OMS 2 para países em desenvolvimento mostra que o consumo energético das crianças estudadas em aleitamento materno exclusivo é adequado (100,6\%) e o das crianças em aleitamento materno complementado fica acima do esperado (156,4\%). Quando os dados de consumo energético são comparados às recomendações da OMS para países desenvolvidos, a adequação diminui para $87,6 \%$ e $141 \%$ para AMEX e AMC, respectivamente. Outras duas recomendações disponíveis apenas para 
aleitamento materno exclusivo 28 , ilustradas na Figura 3, traz como conseqüência déficits maiores de $18,5 \%$ e $8,0 \%$, respectivamente.

Neste estudo, observa-se que os bebês desmamados têm consumo energético superior ao recomendado pela RDA 3, o mesmo acontecendo entre crianças em AMC que tiveram consumo energético superior à recomendação pela OMS 2. A ausência de leite materno ou até mesmo a introdução precoce de alimentos complementares trazem prejuízos à saúde da criança. Estes podem ser diminuição da curva de crescimento quando há oferecimento de alimentos com baixa densidade energética 10 , ou ganho

\section{Resumo}

Com o objetivo de analisar o consumo energético de crianças $\leq$ de 6 meses, em diferentes padrões de aleitamento materno, foram colhidas informações na primeira observação de estudo de coorte. Mães de Campinas, São Paulo, Brasil,foram entrevistadas em seus domicílios sobre: condições sócio-demográficas, características do aleitamento materno, consumo alimentar dos lactentes. A adequação energética foi estimada pelo volume de leite materno consumido com base na equação de regressão de Drewett et al. 1; os valores de referência seguiram recomendação da Organização Mundial da Saúde 2 e da Food and Nutrtition Board 3. A população de estudo (118) caracteriza-se como de classe média baixa, em condições satisfatórias de moradia e saneamento. O desmame inicia-se precocemente, com mediana de duração do aleitamento materno exclusivo de 2,7 meses. O volume de leite materno consumido foi de $561 \mathrm{ml}, 558,9 \mathrm{ml}$ e de $515,2 \mathrm{ml}$, em aleitamento materno exclusivo, aleitamento materno com água elou chá e complementado com outros alimentos, respectivamente. O consumo energético médio foi adequado para as crianças em aleitamento materno exclusivo e acima do recomendado para crianças em amamentação complementada ou desmamadas. ponderal acima do esperado para estatura e idade 9 , com riscos para obesidade e doenças crônico-degenerativas ao longo da vida 29,30, quando são ofertados alimentos complementares ou substitutivos do leite materno de alta densidade energética.

Apesar de se ter usado método de estimativa de consumo de leite materno validado em população rural e de nível social diferente, os resultados aqui obtidos mostram que a escolha do mesmo foi apropriada. Isto porque se observou adequação energética do consumo alimentar dos lactentes estudados com a recomendação da OMS para esta faixa etária 2 .

\section{Colaboradores}

F. F. Nejar elaborou o artigo. A. M. Segall-Corrêa contribuiu na análise e interpretação dos dados, na elaboração do artigo, na revisão crítica da versão final e na elaboração do resumo. M. F. Rea participou da revisão crítica do artigo e da elaboração do abstract. R. P. T. Vianna colaborou na coleta e análise dos dados. G. Panigassi contribuiu na revisão crítica do artigo.

\section{Agradecimentos}

Artigo baseado em informações do projeto Consumo Alimentar e Saúde, processo Fundação de Amparo à Pesquisa do Estado de São Paulo 98/14794-7 que originou a tese de mestrado Característica e Adequação do Consumo Alimentar de Crianças, do Nascimento aos Seis Meses de Idade, e sua Relação com o Aleitamento Materno, Departamento de Medicina Preventiva e Social, Faculdade de Ciências Médicas, Universidade Estadual de Campinas, 2001. 


\section{Referências}

1. Drewett RF, Woolridge MW, Jackson DA, Imong SM, Mangklabruks A, Wongsawasdii L, et al. Relationships between nursing patterns, supplementary food intake and breast-milk intake in a rural Thai population. Early Hum Dev 1989; 20:13-23.

2. World Health Organization. Complementary feeding of young children in developing countries: a review of scientific knowledge. Geneva: World Health Organization; 1998.

3. Food and Nutrition Board. Recommended dietary allowances. $10^{\text {th }}$ Ed. Washington, DC: National Academy Press; 1989.

4. Forman MR. Review of research on the factors associated with choice and duration of infant feeding in less-developed countries. Pediatrics 1984; 74 (4 Pt 2):667-94.

5. Hendricks KM, Badruddin SH. Weaning recommendations: the scientific basis. Nutr Rev 1992; 50:125-33.

6. Jelliffe DB, Jelliffe EFP. Human milk in the modern world. 2nd Ed. Oxford: Oxford University Press; 1978.

7. Victora CG, Vaughan P, Lombardi C. Evidence for protection by breastfeeding against infant death from infectious disease in Brazil. Lancet 1987; 8:319-21.

8. Kramer MS, Chalmers B, Hodnett ED, Sevkovskaya Z, Dzikovich I, Shapiro S, et al. Promotion of breastfeeding intervention trial (PROBIT): a randomized trial in the Republic of Belarus. JAMA 2001; 285:416-20.

9. Lutter C. Recommended length of exclusive breastfeeding age of introduction of complementary foods and the wealing dilemma. Diarrhoeal Diseases Control Programme. Washington DC: World Health Organization; 1992.

10. Cohen RJ, Brown KH, Canahuati J, Rivera LL, Dewey KG. Effects of age of introduction of complementary foods on infant breast milk intake, total energy intake, and growth: a randomise intervention study in Honduras. Lancet 1994; 344: 288-93.

11. Haider R, Islam A, Kabir I, Habte D. Early complementary feeding is associated with low nutrition status of young infants recovering from diarrhoea. J Trop Pediatr 1996; 42:170-2.

12. World Health Organization. Infant and young child nutrition. Geneva: World Health Organization; 2002.

13. Akre J. Alimentação infantil: bases fisiológicas. São Paulo: Rede Internacional em Defesa do Direito de Amamentar; 1994.

14. Monte C, Sá MLB. Guias alimentares para crianças de 6-23 meses no Nordeste do Brasil: da teoria a pratica. Fortaleza: The British Council; 1998.

15. Ministério da Saúde. Guia alimentar para crianças menores de dois anos. Brasília: Ministério da Saúde/Organização Pan-Americana da Saúde; 2002.
16. Vianna RPT. Consumo alimentar e saúde [Tese de Doutorado]. Campinas: Faculdade de Ciências Médicas, Universidade Estadual de Campinas; 2002.

17. Calderon-de-la-Barca AM, Bolanos AV, Caire-Juvera G, Roman-Perez R, Valencia ME, Casanueva E, et al. Evaluacion del consumo de leche humana por dilucion com deutério y deteccion por espectroscopia de infrarrojo. Perinatol Reprod Hum 1998; 12:142-50.

18. Imong SM, Jackson DA, Wongsawasdii L, Ruckphaophunt S, Tansuhaj A, Chiowanich P, et al. Predictors of breast milk intake in rural northern Thailand. J Pediatr Gastroenterol Nutr 1989; 8:359-70.

19. Infante CB, Lara WC, Mardones FS, Rosso PR, Vio FR. Medición de ingesta de líquidos en base a la diluición con deutério. Arch Latinoam Nutr1988; 38:834-43.

20. Organización Mundial de la Salud. Indicadores para evaluar las practicas de lactancia materna. Geneva: Organización Mundial de la Salud/Organización Panamericana de la Salud; 1991.

21. Fundação Instituto Brasileiro de Geografia e Estatística. Contagem populacional, 1996. http:// www.ibge.gov.br (acessado em 17/Jul/2001).

22. Oragnización Mundial de la Salud. Medición del cambio del estado nutricional. Geneva: Organización Mundial de la Salud; 1983.

23. Instituto Nacional de Alimentação e Nutrição. Pesquisa Nacional sobre Saúde Nutrição (PNSN): resultados preliminares. Brasília: Instituto Nacional de Alimentação e Nutrição; 1992.

24. Sociedade Civil Bem-Estar Familiar no Brasil/Demographic and Health Surveys. Pesquisa Nacional sobre Demografia e Saúde (PNDS). Rio de Janeiro: Sociedade Civil Bem-Estar Familiar no Brasil; 1997.

25. Rea MF, Toma TS. Proteção do leite materno e ética. Rev Saúde Pública 2000; 34:388-95.

26. Carvalho M, Robertson S, Merkatz R, Klaus M. Milk intake and frequency of feeding in breast fed infants. Early Hum Dev 1982; 7:155-63.

27. Dewey KG, Heining MJ, Nommsen LA, Lonnerdal B. Maternal versus infant factors related to breast milk intake and residual milk volume: the darling study. Pediatrics 1991; 87:829-37.

28. Butte NF. Energy requirements of infants. Eur J Clin Nutr 1996; 50 Suppl 1:S24-36.

29. Kleinman RE. Complementary feeding and later health. Pediatrics 2000; 106:1287-8.

30. Gimeno SG, Souza JMP. Amamentação ao seio, amamentação com leite de vaca e o diabetes mellitus tipo 1: examinando as evidências. Rev Bras Epidemiol 1998; 1:4-13.

Recebido em $7 /$ Ago/2003

Versão final reapresentada em 13/Jan/2003

Aprovado em 30/Jul/2003 